developing field and we would be interested to learn of other approaches which have contributed to the success of such attachments.

\section{References}

Brown, L. \& Tower, J. (1990) Psychiatrists in primary care: would general practitioners welcome them? Journal of the Royal College of General Practitioners, 40, 369-371. HORDER, J. (1988) Working with general practitioners. The British Journal of Psychiatry 153, 513-521.
Mrtchell, A. (1985) Psychiatrists in primary health care settings. British Journal of Psychiatry, 147, 371-379.

StrathdeE, G. \& Williams, P. (1984) A survey of psychiatrists in primary care: the silent growth of a new service. Journal of the Royal College of General Practitioners, 34, 615-618.

Williams, P. \& WALlACE, B. (1974) General practitioners and psychiatrists do they communicate? British Medical Journal, $i$, 505-507.

A full list of references is available from Dr Strathdee.

\title{
A mothers' group in a child guidance clinic
}

\author{
AnNeTte Goulden, Senior Registrar; and Elund Dorkings, Registrar, The Park \\ Hospital for Children, Headington, Oxford OX3 7LQ
}

We decided to set up a short-term, closed group of mothers attending our local clinic, with the following questions in mind.

Is group therapy helpful for parents of children referred to child guidance clinics? Will they benefit from the therapeutic factors associated with group work: instillation of hope, universality, catharsis, interpersonal learning and group cohesiveness? (Vinogradov \& Yalom, 1989).

Can change be achieved by brief intervention? Research by Benson \& Turk (1989) implied that time limited groups are viable. They ran a 20 month group for anxious and depressed mothers, and sited a positive outcome as a fall in consultation rates, possibly due to a reduction of externalisation of maternal problems onto their children.

How would change be assessed in our group? We predicted that mothers would construe problems differently and that children would change in response to their mothers' altered perceptions and behaviour.

What would mothers wish to discuss? Ruel \& Adams (1981), described 12 themes which became apparent over six months. Would similar themes evolve in our short-term group?

\section{The study}

\section{Group setting}

We selected mothers from the previous 12 months clinics and told each that the group would meet for ten one hour sessions to discuss parenting issues. Members would gain by sharing their problems and by helping others from their own experience, within the context of confidentiality, punctuality and commitment.

\section{Group members}

The nine mothers were aged between 28 and 46 years. One was receiving treatment for depression, another had a history of alcoholism, and a third of drug abuse. Two worked from home and seven were fulltime housewives. Their children ranged in age from 12 to 17 years. Two attended public schools, four went to state schools and three were at college. One had Asperger's syndrome, two were depressed, three had conduct disorder and one child refused to go to school. One child had psychosomatic "fits" and one teenager had agoraphobia.

\section{Structure of the sessions}

At the beginning of each session, we facilitated expression of feelings around the roles of daughter, mother, lover and wife. Towards the end we highlighted a major theme which delivered a clear message for the participants to take home.

\section{Outcome}

\section{Group development}

In the first three sessions members became mutually supportive as they realised that they were not different in their problems as mothers. Discussion concentrated on management of their children's problems.

By the fourth session, the group had stabilised, members sat in the same place each week. They expressed anger towards their husbands and children and towards those seen to be incompetent and uncaring: teachers, psychologists and doctors. This was interpreted as covert anger towards us as imperfect "parental" therapists. 
A realistic awareness of the therapists' role and that of other family members and professionals developed in the eighth session. Previous rivalry for attention was overcome, enabling greater trust and a desire to continue meeting after the group ended.

\section{Group themes}

Isolation

Initially, members felt their problems were unique, and adopted roles such as the questioning therapist, the caring mother, the needy but obstinate adolescent. As common feelings relating to their families and themselves became evident, their sense of isolation diminished. This underlying unity was deepened by the sense of impending loss as the group came to an end.

\section{Mothering}

Each member felt guilty for not being a perfect mother despite expending all her energy on children, husband and even parents, to the detriment of herself. Most denied negative feelings of motherhood, fearing these might harm their children, but reappraisal resulted in the recognition that being a "good enough" mother meant being only $50 \%$ perfect. Children could accept their own bad feelings more readily if their mothers could express and contain such feelings themselves.

\section{Responsibility}

Needing to be perfect mothers, members made themselves responsible for their children's and husband's feelings. Conflict between family responsibility and job satisfaction was realised in the difficulty in setting boundaries between space for themselves, work and family. Taking responsibility for everyone's happiness became a group joke which helped members to recognise their own needs and wishes with less guilt and more honesty.

\section{Self identity}

Members identified with the caring roles of mother, wife, and daughter, but a striking group dress culture of jeans and sloppy tops created an ambience of asexuality, pre-marital freedom and regression to adolescence. They expressed resentment and rejection of what was expected of them in their feminine roles, and questioned what lay beneath those roles and whether they could change.

\section{Anger}

The need to accept the paradox of both loving and resenting their family became apparent. Anger was acted out, but acceptance of ambivalent feelings followed. Members felt less guilty about "bad" feelings in that they were not unique in feeling them and did not destroy the group by expressing them.

\section{Loss}

Members had to recognise their children's own shortcomings and accept the loss of the hoped-for perfect child. Acknowledgement of other losses followed, particularly acceptance of their own less than perfect parents, echoed by the incipient loss of us as therapists. Finally, they shared the reality of loss in death.

\section{Follow-up}

At a ten week follow-up meeting, one member remained psychiatrically ill but the group had carried on meeting without a therapist, and felt that their children posed less of a problem.

\section{Conclusion}

The therapeutic factors of catharsis, universality, mutual support, altruism, interpersonal learning and group cohesiveness operated within our short-term group, allowing feelings to be recognised, expressed and understood. The group produced change in how the mothers felt about themselves and their families, and in the behaviour of their "problem" children.

Such groups can be run by trainees in shortlived posts within a psychiatric training scheme, and provide valuable insight into the process of group therapy.

\section{References}

BENSON, P. \& TURK, T. (1988) Group therapy in a general practice setting for frequent attenders: a controlled study of mothers with preschool children. Journal of the Royal College of General Practitioners, 38, 539-541.

RUEL, A. \& ADAMS, G. (1981) A parenting group in general practice. Journal of the Royal College of General Practitioners, 31, 496-499.

VinogRadov, S. \& Yalom, I. (1989) Group Psychotherapy. Washington and London: American Psychiatric Press Concise Guides. 